\title{
THE EXISTENCE OF PERIODIC AND SUBHARMONIC SOLUTIONS TO SUBQUADRATIC DISCRETE HAMILTONIAN SYSTEMS
}

\author{
ZHAN ZHOU ${ }^{\bullet} 1$, JIANSHE YU ${ }^{1}$ and ZHIMING GUO ${ }^{1}$
}

(Received 22 December, 2003; revised 15 January, 2005)

\begin{abstract}
In this paper, by using critical point theory, we establish some results for the existence of periodic and subharmonic solutions to subquadratic discrete Hamiltonian systems.
\end{abstract}

\section{Introduction}

Let $\mathbb{N}, \mathbb{Z}, \mathbb{R}$ be the set of all natural numbers, integers and real numbers respectively. For $a, b \in \mathbb{Z}$, define $\mathbb{Z}(a)=\{a, a+1, \ldots\}$ and $\mathbb{Z}(a, b)=\{a, a+1, \ldots, b\}$ when $a \leq b$.

Consider the discrete Hamiltonian system

$$
\left\{\begin{array}{l}
\Delta x_{1}(n)=-H_{x_{2}}\left(n, x_{1}(n+1), x_{2}(n)\right), \\
\Delta x_{2}(n)=H_{x_{1}}\left(n, x_{1}(n+1), x_{2}(n)\right),
\end{array}\right.
$$

where $x_{1}, x_{2} \in \mathbb{R}^{d}, H\left(n, x_{1}, x_{2}\right) \in C^{1}\left(\mathbb{R}^{d} \times \mathbb{R}^{d}, \mathbb{B}\right)$ for each $n \in \mathbb{Z}$ and $\Delta x_{i}(n)=$ $x_{i}(n+1)-x_{i}(n), i=1,2$.

Suppose that $H\left(n, x_{1}, x_{2}\right)$ is $m$-periodic in the first variable $n$ where $m$ is a positive integer. We are interested in the existence of $m$-periodic solutions of (1.1). For any positive integer $p$, a $p m$-periodic solution of (1.1), as usual, is also called a subharmonic solution of (1.1).

We may think of system (1.1) as being a discrete analogue of the following Hamiltonian system:

$$
\left\{\begin{array}{l}
d x / d t=-H_{y}(t, x(t), y(t)), \\
d y / d t=H_{x}(t, x(t), y(t)),
\end{array}\right.
$$

'School of Mathematics and Information Sciences, Guangzhou University, Guangzhou 510405 , People's Republic of China; e-mail: zzhou0321@hotmail.com.

(C) Australian Mathematical Society 2005, Serial-fee code 1446-181 1/05 
which has been studied extensively by many scholars. In particular, by using critical point theory, P. H. Rabinowitz, V. Benci, J. Mahwin and K. C. Chang etc. have obtained some significant results for the existence of periodic and subharmonic solutions of (1.2). We refer the reader to $[4,6,12-15]$ and references therein for further details.

Since the behaviour of a discrete system is sometimes sharply different to the behaviour of the corresponding continuous system (see, for example, $[1,16,17]$ ), many scholars have investigated discrete Hamiltonian systems for the disconjugacy, boundary value problems, oscillations and asymptotic behaviour of $(1.1)[3,5,7,8,11]$. Only very few papers deal with the existence of periodic solutions even to general difference equations, see for example $[2,9,16,17]$. As is well known, critical point theory is an important tool for dealing with the existence of periodic solutions of differential equations. It is natural for us to think that critical point theory may be applied to prove the existence of periodic solutions of system (1.1).

In this paper, we will consider the existence of periodic and subharmonic solutions of system (1.1) by using critical point theory.

Let $z=\left(x_{1}^{T}, x_{2}^{T}\right)^{T}$ where $x^{T}$ denotes the transpose of $x, H(n, z)=H\left(n, x_{1}, x_{2}\right)$ and $\nabla_{z} H(n, z)$ represents the gradient of $H(n, z)$ in $z$. For any $k \in \mathbb{N},|\cdot|$ will denote the Euclidean norm in $\mathbb{R}^{k}$ defined by

$$
|x|=\left(\sum_{i=1}^{n} x_{i}^{2}\right)^{1 / 2}, \quad \text { for any } x=\left(x_{1}, x_{2}, \ldots, x_{n}\right)^{T} \in \mathbb{R}^{k}
$$

The main results of this paper are as follows.

THEOREM 1.1. Suppose that $H(n, z)$ satisfies

$\left(\mathrm{H}_{1}\right) \quad H(n, z) \in C\left(\mathbb{R}^{2 d}, \mathbb{R}\right)$ for each $n \in \mathbb{Z}$ and there exists a positive integer $m$ such that for all $(n, z) \in \mathbb{Z} \times \mathbb{R}^{2 d}, H(n+m, z)=H(n, z)$;

$\left(\mathrm{H}_{2}\right)$ there is a constant $M_{0}>0$ such that for all $(n, z) \in \mathbb{Z} \times \mathbb{R}^{2 d},\left|H_{z}(n, z)\right| \leq M_{0}$;

$\left(\mathrm{H}_{3}\right) \quad H(n, z) \rightarrow+\infty$ uniformly for $n \in \mathbb{Z}$ as $|z| \rightarrow \infty$.

Then for any given positive integer $p$, system (1.1) possesses at least one pm-periodic solution.

COROLLARY 1.2. If $H(n, z)$ satisfies $\left(\mathrm{H}_{1}\right)-\left(\mathrm{H}_{2}\right)$, and $\left(\mathrm{H}_{3}\right)$ is replaced by $\left(\mathrm{H}_{4}\right) \quad H(n, z) \rightarrow-\infty$ uniformly for $n \in \mathbb{Z}$ as $|z| \rightarrow \infty$,

then the conclusion of Theorem 1.1 holds.

THEOREM 1.3. Suppose $H(n, z)$ satisfies $\left(\mathrm{H}_{1}\right)$ and

$\left(\mathrm{H}_{5}\right)$ there exist constants $R_{1}>0$ and $\alpha \in(1,2)$ such that for any $(n, z) \in \mathbb{Z} \times$ $\mathbb{R}^{2 d},|z| \geq R_{1}$,

$$
0<z \cdot H_{z}(n, z) \leq \alpha H(n, z)
$$


$\left(\mathrm{H}_{6}\right)$ there exist constants $a_{1}>0, a_{2} \geq 0$ and $\gamma \in(1, \alpha]$ such that

$$
H(n, z) \geq a_{1}|z|^{\gamma}-a_{2}, \quad \forall(n, z) \in \mathbb{Z} \times \mathbb{R}^{2 d} .
$$

Then for any positive integer $p>0$, system (1.1) possesses at least one pm-periodic solution.

REMARK 1.1. By integrating inequality (1.3), we have that

$$
H(n, z) \leq a_{3}|z|^{\alpha}+a_{4}
$$

holds for some positive constants $a_{3}$ and $a_{4}$, which implies that

$$
\lim _{|z| \rightarrow \infty} \frac{H(n, z)}{z^{2}}=0
$$

In this case, $F(n, z)$ is called subquadratic at infinity. When $H(n, z)$ grows superquadratic both at zero and at infinity, we refer to [10] for the existence of periodic solutions of system (1.1).

REMARK 1.2. The $p m$-periodic solutions obtained in Theorem 1.1, Corollary 1.2 and Theorem 1.3 may be constant. On some occasions, we may be more interested in nonconstant periodic solutions. The next theorem establishes such a result.

THEOREM 1.4. Suppose $H(n, z)$ satisfies $\left(\mathrm{H}_{1}\right)$ and

$\left(\mathrm{H}_{7}\right) \quad H(n, 0)=0$, for all $n \in \mathbb{Z}$;

$\left(\mathrm{H}_{8}\right)$ there exists a constant $\alpha \in(1,2)$ such that

$$
0<z \cdot H_{z}(n, z) \leq \alpha H(n, z), \quad \forall(n, z) \in \mathbb{Z} \times \mathbb{R}^{2 d} \text { and }|z| \neq 0 ;
$$

$\left(\mathrm{H}_{9}\right)$ there exist constants $a_{5}>0$ and $\gamma \in(1, \alpha]$ such that

$$
H(n, z) \geq a_{5}|z|^{\gamma}, \quad \forall(n, z) \in \mathbb{Z} \times \mathbb{R}^{2 d} .
$$

Then for any positive integer $p>0$, system (1.1) possesses at least one nonconstant pm-periodic solution.

The remaining part of this paper is organised as follows. In the next section, we will find a functional $F$ such that the critical point of $F$ corresponds to the periodic solution of system (1.1). In Section 3, by using critical point theory, we will prove that $F$ possesses at least one critical point and an illustrative example is also given. 


\section{Some basic lemmas}

Let $S$ be the set of sequences $x=\{x(n)\}_{n \in Z}$, that is,

$$
S=\left\{(x(n)\} \mid x(n)=\left(x_{1}^{T}(n), x_{2}^{T}(n)\right)^{T} \in \mathbb{R}^{2 d}, x_{j}(n) \in \mathbb{B}^{d}, j=1,2, n \in \mathbb{Z}\right\} .
$$

For any $x, y \in S, a, b \in \mathbb{R}, a x+b y$ is defined by

$$
a x+b y \triangleq\{a x(n)+b y(n)\} .
$$

Then $S$ is a vector space.

For any given positive integer $p, m, E_{p m}$ is defined as a subspace of $S$ by

$$
E_{p m}=\{x=\{x(n)\} \in S \mid x(n+p m)=x(n), n \in \mathbb{Z}\} .
$$

We note that $E_{p m}$ can be equipped with the inner product $\langle\cdot, \cdot\rangle_{E_{p m}}$ and norm $\|\cdot\|_{E_{p m}}$ as follows:

$$
\langle x, y\rangle_{E_{p m}}=\sum_{j=1}^{p m}(x(j), y(j)), \quad \forall x, y \in E_{p m}
$$

and

$$
\|x\|_{E_{p m}}=\left(\sum_{j=1}^{p m}|x(j)|^{2}\right)^{1 / 2}, \quad \forall x \in E_{p m},
$$

where $(\cdot, \cdot)$ denotes the usual inner product and $|\cdot|$ denotes the Euclidean norm in $\mathbb{R}^{2 d}$.

We define the functional $F$ on $E_{p m}$ as follows:

$$
F(x)=\frac{1}{2} \sum_{n=1}^{p m}(J \Delta L x(n), x(n))+\sum_{n=1}^{p m} H\left(n-1, x_{1}(n), x_{2}(n-1)\right),
$$

for all $x \in E_{p m}$, where $L x(n)=\left(\begin{array}{c}x_{1}(n) \\ x_{2}(n-1)\end{array}\right), J=\left(\begin{array}{cc}0 & -1 \\ 1 & 0\end{array}\right)$ is the standard $2 d \times 2 d$ sympletic matrix and $I$ is the $d \times d$ identity matrix.

By a simple computation, we can get

$$
\begin{aligned}
F(x) & =\sum_{n=1}^{p m}\left(\Delta x_{1}(n), x_{2}(n)\right)+\sum_{n=1}^{p m} H\left(n, x_{1}(n+1), x_{2}(n)\right) \\
& =-\sum_{n=1}^{p m}\left(x_{1}(n), \Delta x_{2}(n-1)\right)+\sum_{n=1}^{p m} H\left(n-1, x_{1}(n), x_{2}(n-1)\right) .
\end{aligned}
$$

Therefore $F^{\prime}(x)=0$ if and only if for any $n \in Z(1, p m)$,

$$
\left\{\begin{array}{l}
\Delta x_{1}(n)=-H_{x_{2}}^{\prime}\left(n, x_{1}(n+1), x_{2}(n)\right), \\
\Delta x_{2}(n)=H_{x_{1}}^{\prime}\left(n, x_{1}(n+1), x_{2}(n)\right)
\end{array}\right.
$$


where $x \in E_{p m}$.

It is clear now that the problem of finding a $p m$-periodic solution of (1.1) has been reduced to that of seeking the critical point of the functional $F$ defined on $E_{p m}$.

Now we will discuss the existence of a critical point of $F$. To this end, for any $x=\{x(n)\} \in E_{p m}$, where $x(n)=\left(x_{1}^{T}(n), x_{2}^{T}(n)\right)^{T} \in \mathbb{R}^{2 d}$, we assume $x_{j}(n)=$ $\left(x_{j}^{1}(n), x_{j}^{2}(n), \ldots, x_{j}^{d}(n)\right)^{T}$ for $j=1,2$ and let

$$
x^{i}=\left(x_{1}^{i}(1), x_{1}^{i}(2), \ldots, x_{1}^{i}(p m), x_{2}^{i}(1), x_{2}^{i}(2), \ldots, x_{2}^{i}(p m)\right)^{T} \in \mathbb{R}^{2 p m} .
$$

Define a map $\phi$ from $E_{p m}$ to $\mathbb{R}^{2 d p m}$ as

$$
\phi(x)=\left(\left(x^{1}\right)^{T},\left(x^{2}\right)^{T}, \ldots,\left(x^{d}\right)^{T}\right)^{T} .
$$

Then $\phi$ is a linear homeomorphism and $\|x\|_{E_{p m}}=|\phi(x)|$.

By (2.4), we have

$$
\begin{aligned}
F(x) & =\sum_{i=1}^{d} \sum_{n=1}^{p m}\left(x_{1}^{i}(n+1)-x_{1}^{i}(n)\right) x_{2}^{i}(n)+\sum_{n=1}^{p m} H\left(n-1, x_{1}(n), x_{2}(n-1)\right) \\
& =\frac{1}{2}(A \phi(x), \phi(x))+\sum_{n=1}^{p m} H\left(n-1, x_{1}(n), x_{2}(n-1)\right), \\
A & =\left(\begin{array}{lll}
B & & 0 \\
& \ddots & \\
0 & & B
\end{array}\right)_{2 d p m \times 2 d p m} \text { and } B=\left(\begin{array}{cc}
0 & Q^{T} \\
Q & 0
\end{array}\right)_{2 p m \times 2 p m},
\end{aligned}
$$

where

$$
Q=\left(\begin{array}{cccccc}
-1 & 1 & 0 & \ldots & 0 & 0 \\
0 & -1 & 1 & \ldots & 0 & 0 \\
0 & 0 & -1 & \ldots & 0 & 0 \\
\ldots & \ldots & \ldots & \ldots & \ldots & \ldots \\
0 & 0 & 0 & \ldots & -1 & 1 \\
1 & 0 & 0 & \ldots & 0 & -1
\end{array}\right)_{p m \times p m}
$$

It is easy to see that $\lambda$ is an eigenvalue of $B$ if and only if $\lambda^{2}$ is an eigenvalue of $Q^{T} Q$ since

$$
Q^{T} Q=\left(\begin{array}{cccccc}
2 & -1 & 0 & \cdots & 0 & -1 \\
-1 & 2 & -1 & \cdots & 0 & 0 \\
0 & -1 & 2 & \cdots & 0 & 0 \\
\cdots & \cdots & \cdots & \cdots & \cdots & \cdots \\
0 & 0 & 0 & \cdots & 2 & -1 \\
-1 & 0 & 0 & \cdots & -1 & 2
\end{array}\right)_{p m \times p m}
$$


is positive semi-definite and $Q^{T} Q$ has the eigenvalue 0 and $p m-1$ other positive' eigenvalues denoted by $0, \lambda_{1}^{2}, \lambda_{2}^{2}, \ldots, \lambda_{p m-1}^{2}$, where $0<\lambda_{1} \leq \lambda_{2} \leq \cdots \leq \lambda_{p m-1}$. Then the eigenvalues of $A$ can be denoted by $0, \lambda_{ \pm 1}, \lambda_{ \pm 2}, \ldots, \lambda_{ \pm p m-1}$ with multiplicity $2 d$ of 0 , where $\lambda_{-j}=-\lambda_{j}$. Let $A^{0}, A^{+}, A^{-}$denote the eigenspace associated with 0 , all positive eigenvalues and all negative eigenvalues respectively, and

$$
E_{p m}^{0}=\phi^{-1}\left(A^{0}\right), \quad E_{p m}^{+}=\phi^{-1}\left(A^{+}\right) \quad \text { and } \quad E_{p m}^{-}=\phi^{-1}\left(A^{-}\right) .
$$

Then $E_{p m}^{0}=\left\{x \in E_{p m} \mid x=\{v\}, v \in \mathbb{R}^{2 d}\right\}$ and $E_{p m}=E_{p m}^{0} \oplus E_{p m}^{+} \oplus E_{p m}^{-}$.

For any $x=\left(x_{1}, x_{2}, \ldots, x_{2 p m}\right)^{T} \in \mathbb{R}^{2 d p m}$, and $r>1$, define

$$
|x|_{r}=\left(\sum_{i=1}^{2 d p m} x_{i}^{r}\right)^{1 / r} \text {. }
$$

Then $|\cdot|_{r}$ is a norm in $\mathbb{R}^{2 d p m}$. We can equip $E_{p m}$ with the norm $\|x\|_{r}$ deduced by

$$
\|x\|_{r}=|\phi(x)|_{r}, \quad \forall x \in E_{p m} .
$$

Clearly, $\|x\|_{2}=\|x\|_{E_{p m}}$. Since $E_{p m}$ is equivalent to the finite-dimensional Hilbert space $\mathbb{R}^{2 d p m},\|\cdot\|_{2}$ and $\|\cdot\|_{r}$ are equivalent, that is, there exist constants $C_{1 r}, C_{2 r}$ such that $C_{2 r} \geq C_{1 r}>0$, and

$$
C_{1 r}\|x\|_{r} \leq\|x\|_{2} \leq C_{2 r}\|x\|_{r}, \quad \forall x \in E_{p m} .
$$

On the other hand, we have

$$
\begin{aligned}
& \lambda_{1}\|u\|_{2}^{2} \leq\langle A \phi(u), \phi(u)\rangle \leq \lambda_{p m-1}\|u\|_{2}^{2}, \quad \text { for } u \in E_{p m}^{+}, \\
& -\lambda_{p m-1}\|v\|_{2}^{2} \leq\langle A \phi(v), \phi(v)\rangle \leq-\lambda_{1}\|v\|_{2}^{2}, \quad \text { for } v \in E_{p m}^{-} \text {, } \\
& \langle A \phi(w), \phi(w)\rangle=0, \quad \text { for } w \in E_{p m}^{0} .
\end{aligned}
$$

Let $X$ be a real Banach space, $l \in C^{1}(X, \mathbb{R})$, that is, $I$ is a continuously Fréchet differentiable functional defined on $X$. The functional $I$ is said to satisfy the PalaisSmale condition (P-S condition) if any sequence $\left\{u_{n}\right\} \subset X$ for which $\left\{I\left(u_{n}\right)\right\}$ is bounded and $l^{\prime}\left(u_{n}\right) \rightarrow 0(n \rightarrow \infty)$ possesses a convergent subsequence in $X$.

Let $B_{r}$ denote the open ball in $X$ about 0 of radius $r$ and let $\partial B_{r}$ denote its boundary.

LEMMA 2.1 (Saddlepoint theorem (see [15])). Let $X$ be a real Banach space, $X=X_{1} \oplus X_{2}$, where $X_{1} \neq\{0\}$ and is finite dimensional. Suppose $I \in C^{\mathrm{l}}(X, \mathbb{R})$ satisfies the $P$-S condition and

$\left(\mathrm{I}_{1}\right)$ there exist constants $\sigma, \rho>0$ such that $I_{\partial B_{\rho} \cap X_{1}} \leq \sigma$, and

$\left(\mathrm{I}_{2}\right)$ there exist $e \in B_{\rho} \cap X_{1}$ and a constant $\omega>\sigma$ such that $\left.I\right|_{e+X_{2}} \geq \omega$.

Then $I$ possesses a critical value $c \geq \omega$ and $c=\inf _{h \in \Gamma} \max _{u \in B_{\rho} \cap X_{1}} l(h(u))$, where $\Gamma=\left\{h \in C\left(\bar{B}_{\rho} \cap X_{1}, X\right)|h|_{\partial B_{\rho} \cap X_{1}}=\mathrm{id}\right)$. 


\section{Proofs of the main results}

PROOF OF TheOREM 1.1. Let $F(x)$ be defined as in (2.3). Clearly, $F \in C^{1}\left(E_{p m}, \mathbb{R}\right)$. In view of $\left(\mathrm{H}_{2}\right)$, there exists a constant $M_{1}>0$ such that

$$
|H(n, z)| \leq M_{1}+M_{0}|z|, \quad \forall(n, z) \in \mathbb{Z} \times \mathbb{R}^{m} .
$$

We will first show that $F$ satisfies the P-S condition. In fact, suppose that $\left\{x^{(k)}\right\}$ is a sequence in $E_{p m}$ such that for any $k \in \mathbb{Z}(1),\left|F\left(x^{(k)}\right)\right| \leq M_{2}$ for some positive constant $M_{2}$ and $F^{\prime}\left(x^{(k)}\right) \rightarrow 0$ as $k \rightarrow \infty$, then for sufficiently large $k,\left|\left\langle F^{\prime}\left(x^{(k)}\right), x\right\rangle\right| \leq\|x\|_{2}$.

Let $x^{(k)}=y^{(k)}+w^{(k)}$, where $y^{(k)}=u^{(k)}+v^{(k)} \in E_{p m}^{+} \oplus E_{p m}^{-}$and $w^{(k)} \in E_{p m}^{0}$. According to (2.6) and the periodicity of $H$, we have

$$
\left\langle F^{\prime}\left(x^{(k)}\right), x\right\rangle=\left\langle A \phi\left(x^{(k)}\right), \phi(x)\right\rangle+\sum_{n=1}^{p m} H_{z}\left(n-1, L x^{(k)}(n)\right) \cdot L x(n), \quad \forall x \in E_{p m} .
$$

Then for sufficiently large $k$,

$$
\begin{aligned}
\left|\left\langle A \phi\left(x^{(k)}\right), \phi\left(u^{(k)}\right)\right\rangle\right| & \leq \sum_{n=1}^{p m}\left|H_{z}\left(n-1, L x^{(k)}(n)\right) \cdot L u^{(k)}(n)\right|+\left\|u^{(k)}\right\|_{2} \\
& \leq M_{0} \sum_{n=1}^{p m}\left|L u^{(k)}(n)\right|+\left\|u^{(k)}\right\|_{2} \leq\left(M_{0} \sqrt{p m}+1\right)\left\|u^{(k)}\right\|_{2}
\end{aligned}
$$

and according to (2.9), we get

$$
\left|\left\langle A \phi\left(x^{(k)}\right), \phi\left(u^{(k)}\right)\right\rangle\right|=\left|\left\langle A \phi\left(u^{(k)}\right), \phi\left(u^{(k)}\right)\right\rangle\right| \geq \lambda_{1}\left\|u^{(k)}\right\|_{2}^{2} .
$$

Thus we have $\lambda_{1}\left\|u^{(k)}\right\|_{2}^{2} \leq\left(M_{0} \sqrt{p m}+1\right)\left\|u^{(k)}\right\|_{2}$, which implies that $\left\{u^{(k)}\right\}$ is bounded.

Similarly, we can show that $\left\{v^{(k)}\right\}$ is bounded. So $\left\{y^{(k)}\right\}$ is bounded.

Next, we need to prove that $\left\{w^{(k)}\right\}$ is bounded. In fact,

$$
\begin{aligned}
F\left(x^{(k)}\right)= & \frac{1}{2}\left\langle A \phi\left(x^{(k)}\right), \phi\left(x^{(k)}\right)\right\rangle+\sum_{n=1}^{p m} H\left(n-1, L x^{(k)}(n)\right) \\
= & \frac{1}{2}\left\langle A \phi\left(y^{(k)}\right), \phi\left(y^{(k)}\right)\right\rangle+\sum_{n=1}^{p m} H\left(n-1, L w^{(k)}(n)\right) \\
& +\sum_{n=1}^{p m}\left[H\left(n-1, L x^{(k)}(n)\right)-H\left(n-1, L w^{(k)}(n)\right)\right] .
\end{aligned}
$$


So,

$$
\begin{aligned}
& \left|\sum_{n=1}^{p m} H\left(n-1, L w^{(k)}(n)\right)\right| \\
& \quad \leq\left|F\left(x^{(k)}\right)\right|+\frac{1}{2}\left|\left\langle A \phi\left(y^{(k)}\right), \phi\left(y^{(k)}\right)\right\rangle\right| \\
& \quad+\sum_{n=1}^{p m}\left|H\left(n-1, L x^{(k)}(n)\right)-H\left(n-1, L w^{(k)}(n)\right)\right| \\
& \leq M_{2}+\frac{1}{2} \lambda_{p m-1}\left|\phi\left(y^{(k)}\right)\right|^{2}+\sum_{n=1}^{p m}\left|H_{z}\left(n-1, L\left(w^{(k)}(n)+\theta y^{(k)}(n)\right)\right)\right|\left|L y^{(k)}(n)\right| \\
& \leq M_{2}+\frac{1}{2} \lambda_{p m-1}\left\|y^{(k)}\right\|_{2}^{2}+M_{0} \sqrt{p m}\left\|y^{(k)}\right\|_{2},
\end{aligned}
$$

where $\theta \in(0,1)$. This implies that $\left\{\sum_{n=1}^{p m} H\left(n-1, L w^{(k)}(n)\right)\right\}$ is bounded.

By Assumption $\left(\mathrm{H}_{3}\right)$, we have that $\left\{w^{(k)}\right\}$ is bounded. If otherwise, there is no harm in assuming that $\left\|w^{(k)}\right\|_{2} \rightarrow \infty$ as $k \rightarrow \infty$. Since there exist $z^{(k)} \in \mathbb{R}^{2 d}, k \in \mathbb{Z}(1)$, such that $w^{(k)}=\left\{z^{(k)}\right\} \in E_{p m}$, then

$$
\left\|w^{(k)}\right\|=\left(\sum_{n=1}^{p m}\left|w^{(k)}(n)\right|^{2}\right)^{1 / 2}=\left(\sum_{n=1}^{p m}\left|z^{(k)}\right|^{2}\right)^{1 / 2}=\sqrt{p m}\left|z^{(k)}\right| \stackrel{k \rightarrow \infty}{\longrightarrow} \infty .
$$

Since $H\left(n-1, L w^{(k)}(n)\right)=H\left(n-1, z^{(k)}\right)$, we have $H\left(n-1, L w^{(k)}(n)\right) \rightarrow+\infty$ as $k \rightarrow \infty$. This contradicts the fact that $\left\{\sum_{n=1}^{p m} H\left(n-1, L w^{(k)}(n)\right)\right\}$ is bounded.

Now we will check that the conditions $\left(I_{1}\right)$ and $\left(I_{2}\right)$ in the Saddlepoint theorem hold. To this end, let $X_{1}=E_{p m}^{-}$and $X_{2}=E_{p m}^{+} \oplus E_{p m}^{0}$. For any $x=u+w \in E_{p m}^{+} \oplus E_{p m}^{0}=$ $X_{2}$, there exist $z^{*} \in \mathbb{R}^{2 d}$ such that $w(n)=z^{*}, \forall n \in \mathbb{Z}$. Thus

$$
\begin{aligned}
F(x)= & \frac{1}{2}\langle A \phi(x), \phi(x)\rangle+\sum_{n=1}^{p m} H(n-1, L x(n)) \\
= & \frac{1}{2}\langle A \phi(u), \phi(u)\rangle+\sum_{n=1}^{p m}(H(n-1, L x(n))-H(n-1, L w(n))) \\
& +\sum_{n=1}^{p m} H(n-1, L w(n)) \\
\geq & \frac{1}{2} \lambda_{1}\|u\|_{2}^{2}+\sum_{n=1}^{p m} H_{z}(n-1, L w(n)+\theta L u(n)) \cdot L u(n)+\sum_{n=1}^{p m} H\left(n-1, z^{*}\right) \\
\geq & \frac{1}{2} \lambda_{1}\|u\|_{2}^{2}-M_{0} \sum_{n=1}^{p m}|L u(n)|+\sum_{n=1}^{p m} H\left(n-1, z^{*}\right)
\end{aligned}
$$




$$
\begin{aligned}
& \geq \frac{1}{2} \lambda_{1}\|u\|_{2}^{2}-M_{0} \sqrt{p m}\left(\sum_{n=1}^{p m}|L u(n)|^{2}\right)^{1 / 2}+\sum_{n=1}^{p m} H\left(n-1, z^{*}\right) \\
& =\frac{1}{2} \lambda_{1}\|u\|_{2}^{2}-M_{0} \sqrt{p m}\|u\|_{2}+\sum_{n=1}^{p m} H\left(n-1, z^{*}\right),
\end{aligned}
$$

where $\theta \in(0,1)$. According to $\left(\mathrm{H}_{3}\right)$, it is easy to see that $H(n, z)$ is bounded from below for $n \in \mathbb{Z}$ and $z \in \mathbb{R}^{2 d}$. That is, $H(n, z) \geq M_{3}$, for all $(n, z) \in \mathbb{Z} \times \mathbb{R}^{2 d}$ for some constant $M_{3}$. Thus

$$
\begin{aligned}
F(x) & \geq \frac{1}{2} \lambda_{1}\|u\|_{2}^{2}-M_{0} \sqrt{p m}\|u\|_{2}+p m M_{3} \\
& =\frac{1}{2} \lambda_{1}\left(\|u\|_{2}-\frac{M_{0}}{\lambda_{1}} \sqrt{p m}\right)^{2}-\frac{M_{0}^{2}}{2 \lambda_{1}} p m+p m M_{3} \geq p m\left(M_{3}-\frac{M_{0}^{2}}{2 \lambda_{1}}\right) .
\end{aligned}
$$

Take $\omega=p m\left(M_{3}-M_{0}^{2} / 2 \lambda_{1}\right)$, then we have

$$
F(x) \geq \omega, \quad \forall x \in X_{2}
$$

Let $e=0$, then $\left(\mathrm{I}_{2}\right)$ holds.

For any $v \in X_{1}=E_{p m}^{-}$, according to (3.1), we have

$$
\begin{aligned}
F(v) & =\frac{1}{2}\langle A \phi(v), \phi(v)\rangle+\sum_{n=1}^{p m} H(n-1, L v(n)) \\
& \leq-\frac{1}{2} \lambda_{1}\|v\|_{2}^{2}+\sum_{n=1}^{p m}\left(M_{0}|L v(n)|+M_{1}\right) \\
& \leq-\frac{1}{2} \lambda_{1}\|v\|_{2}^{2}+M_{0} \sqrt{p m}\|v\|_{2}+p m M_{1} .
\end{aligned}
$$

This implies that $F(v) \rightarrow-\infty$ as $\|v\|_{2} \rightarrow \infty$. Let $\sigma=\omega-1$, then there exists a sufficiently large $\rho>0$ such that

$$
F(v) \leq \sigma, \quad \forall v \in X_{1}, \text { and }\|v\|_{2}=\rho
$$

Thus $\left(I_{1}\right)$ is satisfied. By the Saddlepoint theorem, the proof of Theorem 1.1 is complete.

Proof of Corollary 1.2. Let $G(x)=-F(x), X_{1}=E_{p m}^{+}$and $X_{2}=E_{p m}^{-} \oplus E_{p m}^{0}$. By a similar argument to the proof of Theorem 1.1, we can prove Corollary 1.2.

Proof OF ThEOREM 1.3. First, we need to show that $F$ satisfies the P-S condition. 
Clearly, $F \in C^{1}\left(E_{p m}, \mathbb{R}\right)$. Let $x^{(k)} \in E_{p m}, k \in \mathbb{Z}(1)$ be such that $\left\{F\left(x^{(k)}\right)\right\}$ is bounded and $F^{\prime}\left(x^{(k)}\right) \rightarrow 0$ as $k \rightarrow \infty$. Then there exists a constant $M_{4}>0$ and $k_{0} \in \mathbb{Z}(1)$ such that $\left|F\left(x^{(k)}\right)\right| \leq M_{4}$ for $k \in \mathbb{Z}(1)$ and $\left|\left\langle F^{\prime}\left(x^{(k)}\right), x\right\rangle\right| \leq\|x\|_{2}$ for $k \in \mathbb{Z}\left(k_{0}\right), x \in E_{p m}$.

Since

$$
\left\langle F^{\prime}\left(x^{(k)}\right), x^{(k)}\right\rangle=\left\langle A \phi\left(x^{(k)}\right), \phi\left(x^{(k)}\right)\right\rangle+\sum_{n=1}^{p m} H_{z}\left(n-1, L x^{(k)}(n)\right) \cdot L x^{(k)}(n),
$$

we see that, for $k \in \mathbb{Z}\left(k_{0}\right)$,

$$
\begin{aligned}
M_{4}+\frac{1}{2}\left\|x^{(k)}\right\|_{2} & \geq F\left(x^{(k)}\right)-\frac{1}{2}\left\langle F^{\prime}\left(x^{(k)}\right), x^{(k)}\right) \\
& =\sum_{n=1}^{p m}\left[H\left(n-1, L x^{(k)}(n)\right)-\frac{1}{2} L x^{(k)}(n) \cdot H_{z}\left(n-1, L x^{(k)}(n)\right)\right] .
\end{aligned}
$$

For any $k \in \mathbb{Z}\left(k_{0}\right)$, denote

$S_{1}^{k}=\left\{n \in \mathbb{Z}(1, p m)|| x^{(k)}(n) \mid \geq R_{1}\right\} \quad$ and $\quad S_{2}^{k}=\left\{n \in \mathbb{Z}(1, p m)|| x^{(k)}(n) \mid<R_{1}\right\}$.

Then $S_{1}^{k} \cup S_{2}^{k}=\mathbb{Z}(1, p m)$ and

$$
\begin{aligned}
M_{4}+\frac{1}{2}\left\|x^{(k)}\right\|_{2} \geq & \sum_{n=1}^{p m} H\left(n-1, L x^{(k)}(n)\right)-\frac{1}{2} \sum_{n \in S_{1}^{k}} L x^{(k)}(n) \cdot H_{z}\left(n-1, L x^{(k)}(n)\right) \\
& -\frac{1}{2} \sum_{n \in S_{2}^{k}} L x^{(k)}(n) \cdot H_{z}\left(n-1, L x^{(k)}(n)\right) .
\end{aligned}
$$

In view of (1.3), we have

$$
\begin{aligned}
M_{4}+\frac{1}{2}\left\|x^{(k)}\right\|_{2} \geq & \sum_{n=1}^{p m} H\left(n-1, L x^{(k)}(n)\right)-\frac{\alpha}{2} \sum_{n \in S_{1}^{k}} H\left(n-1, L x^{(k)}(n)\right) \\
& -\frac{1}{2} \sum_{n \in S_{2}^{k}} L x^{(k)}(n) \cdot H_{z}\left(n-1, L x^{(k)}(n)\right) \\
= & \left(1-\frac{\alpha}{2}\right) \sum_{n=1}^{p m} H\left(n-1, L x^{(k)}(n)\right) \\
& +\frac{1}{2} \sum_{n \in S_{2}^{t}}\left[\alpha H\left(n-1, L x^{(k)}(n)\right)-L x^{(k)}(n) \cdot H_{z}\left(n-1, L x^{(k)}(n)\right)\right] .
\end{aligned}
$$


Since $\alpha H(n-1, z)-z \cdot H_{z}(n-1, z)$ is continuous with respect to $z \in \mathbb{R}^{2 d}$ for each $n \in \mathbb{Z}$, there exists a constant $M_{5}>0$ such that $\left|\alpha H(n-1, z)-z \cdot H_{z}(n-1, z)\right| \leq M_{5}$, for all $z \in \mathbb{R}^{2 d}$ and $|z| \leq R_{1}, n \in \mathbb{Z}(1, p m)$. Thus

$$
M_{4}+\frac{1}{2}\left\|x^{(k)}\right\|_{2} \geq\left(1-\frac{\alpha}{2}\right) \sum_{n=1}^{p m} H\left(n-1, L x^{(k)}(n)\right)-\frac{1}{2} p m M_{5} .
$$

By (1.4) and (2.8), we get

$$
\begin{aligned}
M_{4}+\frac{1}{2}\left\|x^{(k)}\right\|_{2} & \geq\left(1-\frac{\alpha}{2}\right) a_{1} \sum_{n=1}^{p m}\left|L x^{(k)}(n)\right|^{\gamma}-\left(1-\frac{\alpha}{2}\right) a_{2} p m-\frac{1}{2} p m M_{5} \\
& \geq\left(1-\frac{\alpha}{2}\right) a_{1}\left(\frac{1}{C_{2 \gamma}}\right)^{\gamma}\left\|x^{(k)}\right\|_{2}^{\gamma}-M_{6},
\end{aligned}
$$

where $M_{6}=(1-\alpha / 2) a_{2} p m+\left(p m M_{5}\right) / 2$.

That is,

$$
\left(1-\frac{\alpha}{2}\right) a_{1}\left(\frac{1}{C_{2 \gamma}}\right)^{\gamma}\left\|x^{(k)}\right\|_{2}^{\gamma}-\frac{1}{2}\left\|x^{(k)}\right\|_{2} \leq M_{4}+M_{6} .
$$

Because $\gamma \in(1,2)$, we see that $\left\{\left\|x^{(k)}\right\|_{2}\right\}$ is bounded. Since $E_{p m}$ is finite dimensional, $\left\{x^{(k)}\right\}$ has a subsequence which is convergent in $E_{p m}$. Therefore $F$ satisfies the P-S condition.

Now we prove that $F$ satisfies $\left(\mathrm{I}_{1}\right)$ and $\left(\mathrm{I}_{2}\right)$. To this end, let $X_{1}=E_{p m}^{-}$and $X_{2}=E_{p m}^{+} \oplus E_{p m}^{0}$. Then for any $x=u+w \in E_{p m}^{+} \oplus E_{p m}^{0}=X_{2}$,

$$
\begin{aligned}
F(x) & =\frac{1}{2}\langle A \phi(u), \phi(u)\rangle+\sum_{n=1}^{p m} H(n-1, L x(n)) \\
& \geq \frac{1}{2} \lambda_{1}\|u\|_{2}^{2}+\sum_{n=1}^{p m}\left(a_{1}|L x(n)|^{\gamma}-a_{2}\right) . \\
& \geq-a_{2} p m .
\end{aligned}
$$

Let $\omega=-a_{2} p m, e=0$ and $\sigma=\omega-1$. Then $F$ satisfies $\left(\mathrm{I}_{2}\right)$. By $(2.10)$, for $v \in X_{1}=E_{p m}^{-}$, according to (1.5),

$$
\begin{aligned}
F(x) & =\frac{1}{2}\langle A \phi(v), \phi(v)\rangle+\sum_{n=1}^{p m} H(n-1, L v(n)) \\
& \leq-\frac{1}{2} \lambda_{1}\|v\|_{2}^{2}+a_{3} \sum_{n=1}^{p m}|L v(n)|^{\alpha}+a_{4} p m \\
& \leq-\frac{1}{2} \lambda_{1}\|v\|_{2}^{2}+a_{3}\left(\frac{1}{C_{1 \alpha}}\right)^{\alpha}\|v\|_{2}^{\alpha}+a_{4} p m .
\end{aligned}
$$


Since $1<\alpha<2$, there exists a sufficiently large constant $\rho>0$ such that $F(v) \leq \sigma$ for all $v \in X_{1}$ and $\|v\|=\rho$. Thus $\left(\mathrm{I}_{1}\right)$ holds. According to the Saddlepoint theorem, there exists at least one critical point of $F$. And the proof is complete.

PROOF OF THEOREM 1.4. According to the proof of Theorem 1.3, we know that the functional $F \in C^{1}\left(E_{p m}, \mathbb{R}\right)$ has a critical value $c$ and (1.1) has a corresponding solution. By $\left(\mathrm{H}_{7}\right)$, we see that the zero solution is a constant solution of system (1.1). If $x(n) \equiv z \in \mathbb{R}^{2 d}$ is another constant solution of system (1.1), then $H_{x_{1}}(n, z)=0$ and $H_{x_{2}}(n, z)=0$. This implies that $H_{z}(n, z)=0$ for $n \in \mathbb{Z}$. By (1.6), we have $z=0$. Thus the zero solution is the only constant solution of system (1.1). Since $F(0)=0$, to complete the proof it suffices to show that $F$ has a critical value $c>0$. To this end, assume that $\bar{y}_{0} \in \mathbb{R}^{2 d p m}$ is a nonzero eigenvector of $A$ associated with the eigenvalue $-\lambda_{1}$ and with $\left|\bar{y}_{0}\right|=\delta$ small enough, it holds that

$$
\omega=\delta^{\gamma}\left(a_{5}\left(\frac{1}{C_{2 \gamma}}\right)^{\gamma}-\frac{1}{2} \lambda_{1} \delta^{2-\gamma}\right)>0 .
$$

Let $y_{0}=\phi^{-1}\left(\bar{y}_{0}\right) \in E_{p m}^{-}, X_{1}=E_{p m}^{-}$and $X_{2}=y_{0}+E_{p m}^{+} \oplus E_{p m}^{0}$.

For any $x=y_{0}+u+w \in X_{2}$, where $u \in E_{p m}^{+}, w \in E_{p m}^{0}$. According to (2.6), we have

$$
\begin{aligned}
F(x) & =\frac{1}{2}\langle A \phi(x), \phi(x)\rangle+\sum_{n=1}^{p m} H(n-1, L x(n)) \\
& \geq \frac{1}{2}\left\langle A \phi\left(y_{0}\right), \phi\left(y_{0}\right)\right\rangle+\frac{1}{2}\langle A \phi(u), \phi(u)\rangle+a_{5} \sum_{n=1}^{p m}|L x(n)|^{\gamma} \\
& \geq-\frac{1}{2} \lambda_{1}\left\|y_{0}\right\|_{2}^{2}+\frac{1}{2} \lambda_{1}\|u\|_{2}^{2}+a_{5}\left(\frac{1}{C_{2 \gamma}}\right)^{\gamma}\left(\left\|y_{0}\right\|_{2}^{2}+\|u\|_{2}^{2}+\|w\|_{2}^{2}\right)^{\gamma / 2} \\
& \geq-\frac{1}{2} \lambda_{1}\left\|y_{0}\right\|_{2}^{2}+a_{5}\left(\frac{1}{C_{2 \gamma}}\right)^{\gamma}\left\|y_{0}\right\|_{2}^{\gamma} \\
& =\delta^{\gamma}\left(a_{5}\left(\frac{1}{C_{2 \gamma}}\right)^{\gamma}-\frac{1}{2} \lambda_{1} \delta^{2-\gamma}\right)=\omega .
\end{aligned}
$$

This shows that $F$ satisfies the condition $\left(\mathrm{I}_{1}\right)$.

For any $v \in X_{1}$, similar to the proof of Theorem 1.3, we have $F(v) \rightarrow-\infty$ as $\|v\|_{2} \rightarrow \infty$. So, there exists a sufficiently large constant $\rho$ such that $F(v) \leq \omega-1$ for $v \in X_{1},\|v\|=\rho$, which implies that $F$ satisfies the condition $\left(\mathrm{I}_{2}\right)$. Therefore $F$ possesses a critical value $c \geq \omega>0$. The proof of Theorem 1.4 is complete.

Finally, we give an example to illustrate our conclusions. 
EXAMPLE. Consider the system (1.1) with

$$
H\left(n, x_{1}, x_{2}\right)=\left(a\left|x_{1}\right|^{\gamma}+b\left|x_{2}\right|^{\gamma}\right) \psi(n), \quad \forall n \in \mathbb{Z},\left(x_{1}, x_{2}\right)^{T} \in \mathbb{R}^{2},
$$

where $a, b>0, \gamma \in(1,2)$ and $\psi(n)$ is a positive $m$-periodic function.

We now check that all conditions in Theorem 1.4 hold. Clearly, $\left(\mathrm{H}_{1}\right)$ and $\left(\mathrm{H}_{7}\right)$ hold.

Let $z=\left(x_{1}, x_{2}\right)^{T}$ and $\bar{a}=\min \{\psi(n) \mid n \in \mathbb{Z}(1, m)\}>0$. Then

$$
z \cdot H_{z}(n, z)=\left(a \gamma\left|x_{1}\right|^{\gamma}+b \gamma\left|x_{2}\right|^{\gamma}\right) \psi(n)=\gamma H(n, z),
$$

which implies that $\left(\mathrm{H}_{8}\right)$ holds.

At last, we have

$$
\begin{aligned}
H(n, z) & \geq \bar{a} \min \{a, b\}\left(\left|x_{1}\right|^{\gamma}+\left|x_{2}\right|^{\gamma}\right) \\
& \geq \bar{a} \min \{a, b\}\left(\frac{1}{C_{2 \gamma}}\right)^{\gamma}\left(\left|x_{1}\right|^{2}+|x|_{2}^{2}\right)^{\gamma / 2} \\
& =\bar{a} \min \{a, b\}\left(\frac{1}{C_{2 \gamma}}\right)^{\gamma}|z|^{\gamma} .
\end{aligned}
$$

So, $\left(\mathrm{H}_{9}\right)$ holds.

According to Theorem 1.4, we see that, for any given positive integer $p$, the system (1.1) with $H$ as defined in (3.4) possesses at least one nonconstant $p m$-periodic solution.

\section{Acknowledgements}

The project is supported by the Excellent Young Teachers Program of MOE, P. R. C. and the Research Fund for the Doctoral Program of Higher Education of PR China (Grant No. 20020532014).

\section{References}

[1] R. P. Agarwal, Difference equations and inequalities: theory, methods and applications, 2nd ed. (Marcel Dekker, New York, 2000).

[2] R. P. Agarwal and W. Zhang, "Periodic solutions of difference equations with general periodicity", Comput. Math. Appl. 42 (2001) 719-727.

[3] C. D. Ahlbrandt and A. C. Peterson, Discrete Hamiltonian systems (Kluwer Academic Publishers, Dordrecht, 1996).

[4] V. Benci and P. H. Rabinowitz, "Critical point theorems for indefinite functionals", Inventiones Math. 52 (1979) 241-273. 
[5] M. Bohner, "Linear Hamiltonian difference systems: disconjugacy and Jacobi-type conditions", J. Math. Anal. Appl. 199 (1996) 804-826.

[6] K. C. Chang, Critical point theory and its applications (Science and Technical Press, Shanghai, China, 1980).

[7] S. Chen, "Disconjugacy, disfocality, and oscillation of second order difference equations", J. Differential Equations 107 (1994) 383-394.

[8] L. H. Erbe and P. Yan, "Disconjugacy for linear Hamiltonian difference systems", J. Math. Anal. Appl. 167 (1992) 355-367.

[9] Z. Guo and J. Yu, "The existence of periodic and subharmonic solutions for second-order superlinear difference equations", Science in China, Mathematics 46 (2003) 506-515.

[10] Z. Guo and J. Yu, "Periodic and subharmonic solutions for superquadratic discrete Hamiltonian systems", Nonl. Anal., TMA 55 (2003) 969-983.

[11] P. Hartman, "Difference equations: disconjugacy, principal solutions, Green's functions, complete monotonicity", Trans. Amer. Math. Soc. 246 (1978) 1-30.

[12] J. Mawhin and M. Willem, Critical point theory and Hamiltonian systems (Springer, New York, 1989).

[13] P. H. Rabinowitz, "Periodic solutions of Hamiltonian systems", Comm. Pure Appl. Math. 31 (1978) 157-184.

[14] P. H. Rabinowitz, "On subharmonic solutions of Hamiltonian systems", Comm. Pure Appl. Math. 33 (1980)609-633.

[15] P. H. Rabinowitz, Minimax methods in critical point theory with applications to differential equations, CBMS Regional Conference Series in Mathematics 65 (Published for the Conference Board of the Mathematical Sciences, Washington, DC; by Amer. Math. Soc., Providence, RI, 1986).

[16] Z. Zhou and J. Wu, "Attractive periodic orbits in nonlinear delayed discrete-time neural networks with delayed feedback", J. Differential Equations Appl. 8 (2002) 467-483.

[17] Z. Zhou and J. Wu, "Attractive periodic orbits for discrete monotone dynamical systems arising from delayed neural networks", Dyn. Contin. Discrete Impuls. Syst. Ser. B 10 (2003) 95-106. 